\title{
Sample size and sampling method for evaluation of characteristics of the sunflower ${ }^{1}$
}

\author{
Tamanho amostral e método de amostragem de características do girassol
}

\author{
Paulo Sérgio Lima e Silva ${ }^{2 *}$, Luiz Eduardo Barreto dos Santos ${ }^{2}$, Vianney Reinado de Oliveira ${ }^{2}$, Roberto Pequeno \\ de Sousa ${ }^{2}$ e Patrícia Lianny de Oliveira Fernandes ${ }^{2}$
}

\begin{abstract}
In the sunflower, in addition to seed yield, it is of interest to evaluate number of leaves plant ${ }^{-1}$, head diameter, stem diameter and plant height, since these characteristics may be associated with seed yield or lodging. The objective of the work was to determine the optimal sample size (from which the experimental coefficient of variation, $\mathrm{CV}$, can be established) for evaluation of these four characteristics. Fifteen cultivars were evaluated in an experimental design of randomized blocks with ten replications. The characteristics were evaluated using two sampling methods (in a single row of plants or in the two rows from the useable area of each plot) and sample sizes of from two to 18 sampling units, with an interval of two plants. In the two sampling methods, the experimental accuracy, as measured by the CV, increased with increasing sample size. A sample made up of ten sampling units is suitable for assessing the number of leaves, the diameters of the head and stem, and the plant height in cultivars of the sunflower. From this sample size upwards, additional gains in experimental precision are not obtained. This sample size can be used to evaluate plants in a single row or in two rows taken from the useable area of each plot, since there was virtually no difference between sampling methods with regard to experimental accuracy. However, it is most practical and quick to sample in one row of plants.
\end{abstract}

Key words: Helianthus annuus L.. Plant height. Leaf number. Stem diameter. Head diameter.

RESUMO - No girassol, além do rendimento de sementes, existe interesse na avaliação do número de folhas, dos diâmetros do capítulo e do caule e da altura da planta, e porque estas características podem estar associadas com o rendimento de sementes ou com o acamamento. O objetivo do trabalho foi determinar o tamanho ideal da amostra (a partir do qual o coeficiente de variação experimental, CV, se estabiliza) para avaliação das quatro características referidas. Quinze cultivares foram avaliadas no delineamento experimental de blocos ao acaso com dez repetições. As características foram avaliadas com dois métodos de amostragem (em uma só fileira de plantas ou nas duas fileiras da área útil da parcela) e tamanhos amostrais de duas a 18 unidades amostrais, com intervalos de duas plantas. Nos dois métodos de amostragem, a precisão experimental, medida pelo $\mathrm{CV}$, aumentou com o aumento do tamanho da amostra. Uma amostra formada por dez unidades amostrais é adequada para avaliação do número de folhas, dos diâmetros do capítulo e do caule e da altura da planta de cultivares de girassol. A partir deste tamanho amostral não se obtém ganhos adicionais na precisão experimental. Este tamanho amostral pode ser usado com a avaliação de plantas em uma só fileira ou em duas fileiras da área útil da parcela, desde que, com ele praticamente não existiram diferenças entre métodos de amostragem, no que se refere à precisão experimental. Contudo, é mais prático e rápido fazer a amostragem em uma só fileira de plantas.

Palavras-chave: Helianthus annuus L.. Altura da planta. Número de folhas. Diâmetro do caule. Diâmetro do capítulo.

\footnotetext{
*Autor para correspondência

${ }^{1}$ Recebido para publicação em 02/09/2013; aprovado em 24/10/2014

Parte da Monografia de Graduação do segundo autor apresentada na Universidade Federal Rural do Semi-Árido/UFERSA

${ }^{2}$ Departmento de Fitotecnia/UFERSA/CNPq, Caixa Postal 137137, Mossoró-RN, Brasil, 59.600-970, paulosergio@ufersa.edu.br, engduh@ hotmail.com, vianney.reinaldo@gmail.com,rpequeno@ufersa.edu.br, patricia_liany@hotmail.com
} 


\section{INTRODUCTION}

Interest in the sunflower has increased in Brazil with the possibility of its being exploited as a source of biofuel. In many regions of Brazil, the crop is being studied, with a consequent demand for experimental techniques. Except for information on the size and shape of the plot, where the literature is vast, information is lacking on other experimental techniques for the majority of crops including the sunflower. The situation is more serious when it comes to aspects of sampling, although some crops have been addressed as to sample size (CARGNELUTTI FILHO et al., 2010; NUNES, 1998; SILVA; SILVA; CARGNELUTTI FILHO et al., 2008) and sampling methods (GOMES et al., 1963; SILVA et al., 1993, SILVA; SILVA; NUNES, 1998).

In determining plot size, the characteristic evaluated is yield. But there is interest from researchers in other characteristics. As these characteristics are not as important as yield, there is no interest in evaluating them for all those plants used when evaluating yield. This would even be desirable, as the larger the sample size, the higher the experimental precision (LeCLERG; LEONARD; CLARK, 1966). But the larger the sample size, the greater the time and expense spent on sampling. On the other hand, smaller samples may result in less accuracy, and this would be undesirable. Thus, determining ideal sample sizes becomes interesting, especially when the evaluation of characteristics is time-consuming and laborious. Furthermore, certain sampling methods, besides increasing accuracy are more practical than others (GOMES et al., 1963; SILVA et al., 1993, SILVA; SILVA; NUNES, 1998).

Sample size has been determined by various methods (CARGNELUTTI FILHO et al., 2010; CARGNELUTTI FILHO et al., 2011; LEITE et al., 2009; PARANAIBA; MORAIS; FERREIRA, 2009; SILVA; REGO; CECON, 2011;). In the method where the CV is established, a CV is obtained for each sample size. Sample sizes and CV values are arranged as a table, and the optimal sample size is considered to be that after which there are no significant additional increases in precision. Although sometimes receiving criticism for being subjective, this method has been used because it is simple, easy to apply and provides sample sizes consistent with reality (SILVA; SILVA; NUNES, 1998; WOLKOWSKI; REISDORF; BUNDY, 1988;). On the other hand, most other methods, besides being harder to apply, often indicate sample sizes which are overly small or large (CARGNELUTTI FILHO et al., 2010; CARGNELUTTI FILHO et al., 2011; PARANAIBA; MORAIS; FERREIRA, 2009).

In the sunflower, in addition to seed yield, the plant height, stem diameter, number of leaves and head diameter are evaluated, among other characteristics. The first two characteristics are associated with lodging, the number of leaves is associated with the photosynthetic capacity of the plant, and the head diameter is related to seed yield (AMJADIAN; FARSHADFAR; GHOLIPOOR, 2013; JAGADEESAN et al., 2008; YASIN; SINGH, 2010). Several authors (AHMAD; AHMED; TAHIR, 2012; MARTIN et al., 2012) have evaluated these characteristics, reflecting their importance.

The objective of this work was to determine the appropriate sample size (from which the $\mathrm{CV}$ is established) for evaluation of the four above mentioned biometric traits in the sunflower.

\section{MATERIAL AND METHODS}

The work was carried out at the Rafael Fernandes Experimental Farm of the Federal Rural University of the Semi-Arid - UFERSA, from February to May, 2012. This farm is located in the district of Alagoinha, $20 \mathrm{~km}$ away from the town of Mossoró (latitude $5^{\circ} 11^{\prime} \mathrm{S}$, longitude $37^{\circ} 20^{\prime} \mathrm{W}$, at an altitude of $\left.18 \mathrm{~m}\right)$. According to the Gaussen bioclimatic classification, the climate in the Mossoró region is of the 4ath type, severe thermoxerochimenic, being tropical, hot and markedly dry, with a long dry season of seven to eight months and a xerothermic index between 150 and 200. The region has a maximum average air temperature of between 32.1 and $34.5^{\circ} \mathrm{C}$, and an average minimum of between 21.3 and $23.7^{\circ} \mathrm{C}$, with June and July being the coldest months. The average annual rainfall is around $825 \mathrm{~mm}$. Sunlight increases from March to October, with an average of $241.7 \mathrm{~h}$, and the relative air humidity reaches a maximum of $78 \%$ in April and a minimum of $60 \%$ in September (CARMO FILHO; OLIVEIRA, 1989).

The soil of the experimental area is classified as a Red-Yellow Argisol, according to the Brazilian System of Soil Classification (EMPRESA BRASILEIRA DE PESQUISA AGROPECUÁRIA, 2006). After the soil was prepared by harrowing twice, five single soil samples were taken from the experimental area at a depth of $0-20 \mathrm{~cm}$ and combined to form a composite sample. The results of the chemical analysis of the composite sample showed: $\mathrm{pH}\left(\mathrm{H}_{2} \mathrm{O}\right)=6.5$; organic matter $=21 \mathrm{~g} \mathrm{dm}^{-3} ; \mathrm{P}\left(\right.$ Mehlic-1) $=7.1 \mathrm{mg} \mathrm{dm}^{-3} ; \mathrm{K}^{+}$ $=69.2 \mathrm{mg} \mathrm{dm}^{-3} ; \mathrm{Na}^{+}=59.2 \mathrm{mg} \mathrm{dm}{ }^{-3}, \mathrm{Ca}^{2+}=1.4 \mathrm{cmol} \mathrm{dm}^{-3}$; $\mathrm{Mg}^{2+}=0.8 \mathrm{cmol}_{\mathrm{c}} \mathrm{dm}^{-3} ; \mathrm{H}^{+}+\mathrm{Al}^{3+}=0.8 \mathrm{cmol}_{\mathrm{c}} \mathrm{dm}^{-3} ; \mathrm{CEC}$ $=3.5 \mathrm{cmol}_{\mathrm{c}}$.

The soil received as sowing fertilizer $60 \mathrm{~kg} \mathrm{~N}$ (ammonium sulphate), $100 \mathrm{~kg}$ of $\mathrm{P}_{2} \mathrm{O}_{5}$ (superphosphate) and $20 \mathrm{~kg}$ of $\mathrm{K}_{2} \mathrm{O}$ (potassium chloride) per hectare. The nitrogen was applied in three equal parts: at sowing, 20 
days after sowing, and 40 days after sowing. Between the rows a spacing of $0.70 \mathrm{~m}$ was used, with the holes in any one row spaced $0.30 \mathrm{~m}$ apart. Sowing was carried out manually with four seeds per hole. The plants were thinned 20 days after sowing, leaving the largest plant in each hole, and the experiment with a programmed seeding density of 47,619 plants ha $^{-1}$.

A randomized, complete block design was used, with ten replications. The cultivars listed in Table 1, with respective phenologic traits, were evaluated in plots made up of four rows each of $6.0 \mathrm{~m}$ in length. The useable area was considered to be that occupied by the 36 central plants (18 in each row) of the two central rows of each plot. These plants were evaluated when the crop was in full flowering, for number of leaves plant ${ }^{-1}$, head diameter, stem diameter and plant height. Head diameter was measured using a rule. Stem diameter was measured with digital callipers $10 \mathrm{~cm}$ from the ground. Plant height was measured from soil surface to the insertion of the last leaf using a rule. Sample sizes were considered to be 2 to 18 sampling units (plant, for plant height, stem diameter and number of leaves plant $^{-1}$, and head, for head diameter) in intervals of two. Sampling methods considered plants from any one row ("single row") or plants from the two rows ("double row") of the useable area. In the case of the double row, half of the sampling units were evaluated from one row and the remainder from the other. The rows and plants for evaluation were selected with the aid of a random digit table.

The experiment was carried out under rainfed conditions, receiving sprinkler irrigation when necessary, with the experimental plots arranged parallel to the rows of sprinklers. The amount of water required by the sunflower $(5.3 \mathrm{~mm})$ was calculated based on an effective depth of $0.40 \mathrm{~m}$ for the root system. When to irrigate was based on the water retained in the soil at a tension of $0.40 \mathrm{Mpa}$. Irrigation was started after sowing, carried out three times a week, and suspended five days before the heads were harvested. Weeding was carried out by hoe, always assigning the same worker to each block. There were no pest attacks or outbreaks of disease.

The averages of the sampling units for each sample size and sampling method were submitted to variance analysis, with 18 variance analyses being carried out ( 2 methods x 9 sample sizes). Averages for the cultivars were compared at $5 \%$ probability by the Scott-Knott test (1974). The optimal sample size was considered one from which there were no reductions in the coefficient of variation. The variance analyses were done using the SAEG software developed by the Federal University of Viçosa (RIBEIRO JÚNIOR, 2001).

Table 1 - Cultivar type and phenology of the evaluated cultivars

\begin{tabular}{llccc}
\hline \multirow{2}{*}{ Cultivars } & Cultivar type & Beginning of flowerig & Full flowering & Physiological maturity \\
\cline { 3 - 5 } & Three cross & 54 & 56 & 83 \\
\hline Aguará-05 & Single cross & 45 & 48 & 75 \\
Aguará-03 & Single cross & 52 & 56 & 84 \\
Aguará-06 & Single cross & 42 & 44 & 70 \\
Charrua & Single cross & 47 & 51 & 76 \\
Aguará-04 & Single cross & 47 & 54 & 84 \\
Olizun-03 & Variety & 46 & 49 & 76 \\
Embrapa-122 & Single cross & 55 & 56 & 90 \\
Paraíso-65 & Single cross & 42 & 48 & 79 \\
Neon & Single cross & 47 & 52 & 78 \\
BRS-321 & Variety & 47 & 51 & 77 \\
BRS-324 & Single cross & 46 & 48 & 76 \\
Zenit & Single cross & 47 & 50 & 76 \\
Paraíso-55 & Single cross & 49 & 53 & 77 \\
M734 & Single cross & 49 & 54 & 83 \\
Paraíso103CL & & &
\end{tabular}




\section{RESULTS AND DISCUSSION}

For the number of leaves per plant, stem diameter and plant height, the F-test showed effects from the cultivars, at $1 \%$ probability for all sample sizes, with evaluation being carried out on a single row (Table 2) or on both the rows of the useable area of each plot (Table 3). An exception to this finding was seen for stem diameter, evaluated in four plants from both rows, in which differences between cultivars occurred only at $5 \%$ probability (Table 3). However for head diameter, the F-test indicated non-significant effects for the sample sizes of 16, 12 and 6, when the heads were evaluated from a single row (Table 2), and for a sample size of 2, when the heads were evaluated from both rows of the useable area of each plot (Table 3). Sample size in the evaluation of head diameter was apparently not relevant when obtaining those results, provided that no

Table 2 - Summary of variance analysis for characteristics of sunflower cultivars evaluated by sample size in one row of plants from plots of two rows

\begin{tabular}{|c|c|c|c|c|c|c|}
\hline \multirow{3}{*}{$\begin{array}{lr}\text { Sample size } \\
\text { (sampling units) }\end{array}$} & \multirow{3}{*}{$\begin{array}{l}\text { Source of } \\
\text { variation }\end{array}$} & \multirow{3}{*}{$\begin{array}{l}\text { Degrees of } \\
\text { freedom }\end{array}$} & \multicolumn{4}{|c|}{ Characteristics } \\
\hline & & & $\begin{array}{c}\text { Number of leaves } \\
\text { per plant }\end{array}$ & Head diameter & Stem diameter & Plant height \\
\hline & & & \multicolumn{4}{|c|}{ Mean squares ${ }^{1}$} \\
\hline \multirow{4}{*}{18} & Blocks & 9 & 31.1 & 22.0 & 32.4 & 1789.4 \\
\hline & Cultivars & 14 & $83.6^{* *}$ & $3.0^{*}$ & $23.1^{* *}$ & $1668.7 * *$ \\
\hline & Residue & 126 & 3.4 & 1.5 & 2.2 & 96.6 \\
\hline & Blocks & 9 & 30.8 & 18.6 & 38.4 & 1830.7 \\
\hline \multirow{3}{*}{16} & Cultivars & 14 & $91.1 * *$ & $2.8^{\mathrm{ns}}$ & $28.1 * *$ & $1705.0^{* *}$ \\
\hline & Residue & 126 & 3.3 & 1.8 & 2.9 & 99.7 \\
\hline & Blocks & 9 & 36.3 & 19.4 & 36.9 & 1677.0 \\
\hline \multirow{3}{*}{14} & Cultivars & 14 & $97.2 * *$ & $4.6^{* *}$ & $37.0 * *$ & $1954.5^{* *}$ \\
\hline & Residue & 126 & 3.6 & 1.7 & 3.1 & 118.1 \\
\hline & Blocks & 9 & 24.5 & 24.3 & 40.6 & 1982.4 \\
\hline \multirow{3}{*}{12} & Cultivars & 14 & $79.1 * *$ & $2.4^{\mathrm{ns}}$ & $25.5^{* *}$ & $1785.2 * *$ \\
\hline & Residue & 126 & 3.5 & 1.8 & 3.0 & 112.3 \\
\hline & Blocks & 9 & 53.0 & 20.1 & 43.1 & 1711.5 \\
\hline \multirow{3}{*}{10} & Cultivars & 14 & $99.9 * *$ & $3.9^{* * *}$ & $29.6^{* *}$ & $1839.1 * *$ \\
\hline & Residue & 126 & 2.9 & 1.7 & 2.8 & 92.9 \\
\hline & Blocks & 9 & 22.5 & 19.9 & 25.9 & 1644.0 \\
\hline \multirow{3}{*}{8} & Cultivars & 14 & $77.7^{* *}$ & $4.2^{*}$ & $25.6^{* *}$ & $1640.3^{* *}$ \\
\hline & Residue & 126 & 3.6 & 1.9 & 3.0 & 97.5 \\
\hline & Blocks & 9 & 41.9 & 23.4 & 35.5 & 1939.2 \\
\hline \multirow{3}{*}{6} & Cultivars & 14 & $90.5 * *$ & $4.1^{\mathrm{ns}}$ & $27.3 * *$ & $1708.4 * *$ \\
\hline & Residue & 126 & 3.9 & 2.6 & 3.4 & 125.2 \\
\hline & Blocks & 9 & 32.7 & 115.0 & 40.3 & 2162.1 \\
\hline \multirow{3}{*}{4} & Cultivars & 14 & $75.7 * *$ & $8.4^{* * *}$ & $26.5^{* *}$ & $1403.1 * *$ \\
\hline & Residue & 126 & 5.2 & 3.0 & 4.3 & 174.8 \\
\hline & Blocks & 9 & 33.1 & 38.7 & 50.4 & 1632.0 \\
\hline \multirow{2}{*}{2} & Cultivars & 14 & $77.7 * *$ & $10.9^{*}$ & $22.9 * *$ & $1816.5^{* *}$ \\
\hline & Residue & 126 & 9.2 & 5.9 & 5.4 & 153.0 \\
\hline
\end{tabular}

${ }^{1} \mathrm{~ns}, *, * *$ Corresponding non-significant or significant effect at $5 \%$ or at $1 \%$ probability respectively, by the F-test. 
Table 3 - Summary of variance analysis for characteristics of sunflower cultivars evaluated by sample size in two rows of plants from plots of two rows

\begin{tabular}{|c|c|c|c|c|c|c|}
\hline \multirow{3}{*}{$\begin{array}{l}\text { Sample size } \\
\text { (sampling units) }\end{array}$} & \multirow{3}{*}{$\begin{array}{l}\text { Source of } \\
\text { variation }\end{array}$} & \multirow{3}{*}{$\begin{array}{l}\text { Degrees of } \\
\text { freedom }\end{array}$} & \multicolumn{4}{|c|}{ Characteristics } \\
\hline & & & $\begin{array}{c}\text { Number of leaves } \\
\text { per plant }\end{array}$ & Head diameter & Stem diameter & Plant height \\
\hline & & & \multicolumn{4}{|c|}{ Mean squares $^{1}$} \\
\hline \multirow{4}{*}{18} & Blocks & 9 & 26.1 & 24.1 & 37.7 & 1968.1 \\
\hline & Cultivars & 14 & $77.3 * *$ & $3.3^{*}$ & $27.3^{* *}$ & $1762.1^{* *}$ \\
\hline & Residue & 126 & 3.2 & 1.6 & 2.9 & 107.2 \\
\hline & Blocks & 9 & 26.1 & 21.2 & 33.5 & 1850.4 \\
\hline \multirow{3}{*}{16} & Cultivars & 14 & $85.6^{* *}$ & $4.2 * *$ & $24.5 * *$ & $1829.4^{* *}$ \\
\hline & Residue & 126 & 2.9 & 1.6 & 2.5 & 106.9 \\
\hline & Blocks & 9 & 31.4 & 22.7 & 32.8 & 1901.9 \\
\hline \multirow{3}{*}{14} & Cultivars & 14 & $87.7 * *$ & $4.0^{* *}$ & $27.1^{* *}$ & $1774.2^{* *}$ \\
\hline & Residue & 126 & 2.5 & 1.4 & 2.9 & 101.8 \\
\hline & Blocks & 9 & 34.2 & 24.0 & 42.0 & 2043.4 \\
\hline \multirow{3}{*}{12} & Cultivars & 14 & $90.9 * *$ & $3.8 * *$ & $28.7 * *$ & $1813.8^{* *}$ \\
\hline & Residue & 126 & 2.7 & 1.5 & 3.0 & 108.0 \\
\hline & Blocks & 9 & 35.3 & 20.9 & 39.4 & 2278.0 \\
\hline \multirow{3}{*}{10} & Cultivars & 14 & $92.6^{* *}$ & $3.4^{*}$ & $32.9 * *$ & $1672.8^{* *}$ \\
\hline & Residue & 126 & 3.0 & 1.9 & 3.4 & 96.1 \\
\hline & Blocks & 9 & 37.8 & 19.2 & 31.0 & 2077.5 \\
\hline \multirow{3}{*}{8} & Cultivars & 14 & $85.3 * *$ & $5.7^{* * *}$ & $25.0 * *$ & $1743.6^{* *}$ \\
\hline & Residue & 126 & 4.2 & 2.2 & 3.8 & 109.5 \\
\hline & Blocks & 9 & 33.3 & 21.3 & 41.3 & 2189.6 \\
\hline \multirow{3}{*}{6} & Cultivars & 14 & $88.9^{* *}$ & $5.1^{*}$ & $32.2 * *$ & $1756.3^{* *}$ \\
\hline & Residue & 126 & 3.1 & 2.5 & 4.8 & 134.2 \\
\hline & Blocks & 9 & 40.5 & 31.8 & 31.6 & 2446.4 \\
\hline \multirow{3}{*}{4} & Cultivars & 14 & $98.6^{* *}$ & $7.8^{* *}$ & $19.9^{*}$ & $2109.6^{* *}$ \\
\hline & Residue & 126 & 4.4 & 3.1 & 10.8 & 159.3 \\
\hline & Blocks & 9 & 35.4 & 22.2 & 39.7 & 1712.6 \\
\hline \multirow{2}{*}{2} & Cultivars & 14 & $94.0 * *$ & $6.6^{\mathrm{ns}}$ & $37.4 * *$ & $2027.4 * *$ \\
\hline & Residue & 126 & 8.3 & 4.4 & 9.0 & 203.1 \\
\hline
\end{tabular}

${ }^{1} \mathrm{~ns}, *, * *$ Corresponding non-significant or significant effect at $5 \%$ or at $1 \%$ probability respectively, by the F-test.

significant results were observed for samples of between 2 and 18 sampling units. Silva, Silva and Nunes (1998) noted that the F-test indicated no effects from maize cultivars on the number of grains per ear when the sample size varied from 1 to 15 ears and when sampling was carried out randomly or haphazardly. For the length and weight of the cob, the F-test, depending on the sampling method and sample size, showed both non-significant and significant effects from cultivars (at $5 \%$ or $1 \%$ probability) (SILVA; SILVA; NUNES, 1998).
For the number of leaves per plant, stem diameter and plant height, the Scott-Knott test showed differences between cultivars for all sample sizes when measurements were taken from one (Tables 4 and 5) or from two rows of plants (Tables 6 and 7), except for stem diameter, where 4 plants were evaluated (Table 7). However the ScottKnott test (1974) showed no difference between cultivars for head diameter with sample sizes of 18, 16, 12 and 6 (agreeing with the F-test results) when the plants were 
Table 4 - Averages of the number of leaves and of head diameter in sunflower cultivars evaluated by sample size in one row of plants from plots with two rows ${ }^{1}$

\begin{tabular}{|c|c|c|c|c|c|c|c|c|c|}
\hline \multirow{3}{*}{ Cultivar } & \multicolumn{9}{|c|}{ Sample size (number of sampling units) } \\
\hline & 18 & 16 & 14 & 12 & 10 & 8 & 6 & 4 & 2 \\
\hline & \multicolumn{9}{|c|}{ Number of leaves plant ${ }^{-1}$} \\
\hline Aguará-05 & $28.9 \mathrm{a}$ & $28.5 \mathrm{a}$ & $29.0 \mathrm{a}$ & $28.7 \mathrm{a}$ & $29.0 \mathrm{a}$ & $28.4 \mathrm{a}$ & $28.5 \mathrm{a}$ & $28.6 \mathrm{a}$ & $27.8 \mathrm{a}$ \\
\hline Aguará-03 & $27.4 \mathrm{a}$ & $28.0 \mathrm{a}$ & $27.4 \mathrm{a}$ & $27.0 \mathrm{a}$ & $28.0 \mathrm{a}$ & $27.2 \mathrm{a}$ & $28.0 \mathrm{a}$ & $27.6 \mathrm{a}$ & $27.7 \mathrm{a}$ \\
\hline Aguará-06 & $27.4 \mathrm{a}$ & $27.7 \mathrm{a}$ & $27.6 \mathrm{a}$ & $26.8 \mathrm{a}$ & $27.9 \mathrm{a}$ & $27.6 \mathrm{a}$ & $27.4 \mathrm{a}$ & $26.7 \mathrm{a}$ & $27.1 \mathrm{a}$ \\
\hline Charrua & $27.3 \mathrm{a}$ & $26.6 \mathrm{a}$ & $26.7 \mathrm{a}$ & $27.3 \mathrm{a}$ & $26.3 \mathrm{a}$ & $26.6 \mathrm{a}$ & $27.0 \mathrm{a}$ & $27.5 \mathrm{a}$ & $26.3 \mathrm{a}$ \\
\hline Aguará-04 & $26.8 \mathrm{a}$ & $26.9 \mathrm{a}$ & $26.9 \mathrm{a}$ & $26.6 \mathrm{a}$ & $27.5 \mathrm{a}$ & $26.8 \mathrm{a}$ & $26.8 \mathrm{a}$ & $26.8 \mathrm{a}$ & $27.6 \mathrm{a}$ \\
\hline Olizun-03 & $26.7 \mathrm{a}$ & $27.4 \mathrm{a}$ & $27.8 \mathrm{a}$ & $27.0 \mathrm{a}$ & $27.6 \mathrm{a}$ & $26.3 \mathrm{a}$ & $26.9 \mathrm{a}$ & $26.8 \mathrm{a}$ & $26.7 \mathrm{a}$ \\
\hline Embrapa-122 & $24.3 \mathrm{~b}$ & $23.8 \mathrm{~b}$ & $25.0 \mathrm{~b}$ & $25.1 \mathrm{~b}$ & $23.5 \mathrm{~b}$ & $23.6 \mathrm{~b}$ & $22.9 \mathrm{~b}$ & $24.2 \mathrm{~b}$ & $24.4 \mathrm{~b}$ \\
\hline Paraíso-65 & $23.4 \mathrm{~b}$ & $24.3 \mathrm{~b}$ & $23.8 \mathrm{c}$ & $23.1 \mathrm{c}$ & $23.9 \mathrm{~b}$ & $23.5 \mathrm{~b}$ & $24.3 \mathrm{~b}$ & $22.3 \mathrm{c}$ & $24.5 \mathrm{~b}$ \\
\hline Neon & $23.4 \mathrm{~b}$ & $23.5 \mathrm{~b}$ & $22.9 \mathrm{c}$ & $23.1 \mathrm{c}$ & $22.9 \mathrm{~b}$ & $23.4 \mathrm{~b}$ & $24.1 \mathrm{~b}$ & $23.3 \mathrm{~b}$ & $24.8 \mathrm{~b}$ \\
\hline BRS-321 & $22.8 \mathrm{~b}$ & $23.3 \mathrm{~b}$ & $22.2 \mathrm{c}$ & $22.7 \mathrm{c}$ & $22.3 \mathrm{~b}$ & $23.2 \mathrm{~b}$ & $22.0 \mathrm{c}$ & $23.2 \mathrm{~b}$ & $22.6 \mathrm{c}$ \\
\hline BRS-324 & $22.7 \mathrm{~b}$ & $22.4 \mathrm{c}$ & $21.9 \mathrm{c}$ & $22.6 \mathrm{c}$ & $22.2 \mathrm{~b}$ & $22.7 \mathrm{~b}$ & $22.4 \mathrm{~b}$ & $22.2 \mathrm{c}$ & $23.0 \mathrm{c}$ \\
\hline Zenit & $22.0 \mathrm{~b}$ & $22.0 \mathrm{c}$ & $21.9 \mathrm{c}$ & $22.2 \mathrm{c}$ & $22.2 \mathrm{~b}$ & $22.3 \mathrm{~b}$ & $21.5 \mathrm{c}$ & $21.7 \mathrm{c}$ & $21.7 \mathrm{c}$ \\
\hline Paraíso-55 & $21.4 \mathrm{c}$ & $20.8 \mathrm{~d}$ & $20.6 \mathrm{~d}$ & $21.2 \mathrm{~d}$ & $21.1 \mathrm{c}$ & $20.9 \mathrm{c}$ & $20.6 \mathrm{c}$ & $21.8 \mathrm{c}$ & $21.8 \mathrm{c}$ \\
\hline M734 & $20.2 \mathrm{c}$ & $19.9 \mathrm{~d}$ & $20.3 \mathrm{~d}$ & $20.2 \mathrm{~d}$ & $19.5 \mathrm{c}$ & $19.9 \mathrm{c}$ & $20.8 \mathrm{c}$ & $21.0 \mathrm{c}$ & $19.6 \mathrm{c}$ \\
\hline Paraíso103CL & $19.9 \mathrm{c}$ & $19.7 \mathrm{~d}$ & $19.8 \mathrm{~d}$ & $20.3 \mathrm{~d}$ & $20.1 \mathrm{c}$ & $20.2 \mathrm{c}$ & $19.7 \mathrm{c}$ & $20.5 \mathrm{c}$ & $20.2 \mathrm{c}$ \\
\hline Cultivar & \multicolumn{9}{|c|}{ Head diameter $(\mathrm{cm})$} \\
\hline Aguará-06 & $13.1 \mathrm{a}$ & $12.7 \mathrm{a}$ & $13.2 \mathrm{a}$ & $13.0 \mathrm{a}$ & $13.4 \mathrm{a}$ & $12.9 \mathrm{a}$ & $12.5 \mathrm{a}$ & $13.2 \mathrm{a}$ & $13.5 \mathrm{a}$ \\
\hline Zenit & $12.7 \mathrm{a}$ & $12.7 \mathrm{a}$ & $12.6 \mathrm{a}$ & $12.6 \mathrm{a}$ & $12.6 \mathrm{a}$ & $13.2 \mathrm{a}$ & $12.6 \mathrm{a}$ & $12.2 \mathrm{a}$ & $13.2 \mathrm{a}$ \\
\hline Neon & $12.6 \mathrm{a}$ & $12.6 \mathrm{a}$ & $12.6 \mathrm{a}$ & $12.7 \mathrm{a}$ & $12.5 \mathrm{a}$ & $12.7 \mathrm{a}$ & $13.2 \mathrm{a}$ & $12.8 \mathrm{a}$ & $13.1 \mathrm{a}$ \\
\hline Paraíso-65 & $12.6 \mathrm{a}$ & $13.3 \mathrm{a}$ & $13.2 \mathrm{a}$ & $12.3 \mathrm{a}$ & $13.1 \mathrm{a}$ & $13.1 \mathrm{a}$ & $13.3 \mathrm{a}$ & $12.9 \mathrm{a}$ & $14.2 \mathrm{a}$ \\
\hline Paraíso103CL & $12.4 \mathrm{a}$ & $12.5 \mathrm{a}$ & $12.4 \mathrm{a}$ & $12.3 \mathrm{a}$ & $12.3 \mathrm{a}$ & $12.6 \mathrm{a}$ & $12.6 \mathrm{a}$ & $13.2 \mathrm{a}$ & $11.6 \mathrm{~b}$ \\
\hline BRS-321 & $12.4 \mathrm{a}$ & $12.3 \mathrm{a}$ & $11.9 \mathrm{~b}$ & $12.0 \mathrm{a}$ & $12.5 \mathrm{a}$ & $12.3 \mathrm{a}$ & $11.8 \mathrm{a}$ & $13.0 \mathrm{a}$ & $12.6 \mathrm{a}$ \\
\hline Aguará-05 & $12.3 \mathrm{a}$ & $12.5 \mathrm{a}$ & $12.2 \mathrm{~b}$ & $12.8 \mathrm{a}$ & $12.1 \mathrm{~b}$ & $11.9 \mathrm{~b}$ & $12.4 \mathrm{a}$ & $12.2 \mathrm{a}$ & $12.0 \mathrm{~b}$ \\
\hline Aguará-03 & $12.1 \mathrm{a}$ & $12.0 \mathrm{a}$ & $12.2 \mathrm{~b}$ & $11.9 \mathrm{a}$ & $12.0 \mathrm{~b}$ & $11.7 \mathrm{~b}$ & $11.8 \mathrm{a}$ & $11.2 \mathrm{~b}$ & $12.8 \mathrm{a}$ \\
\hline Aguará-04 & $12.1 \mathrm{a}$ & $12.5 \mathrm{a}$ & $12.7 \mathrm{a}$ & $12.3 \mathrm{a}$ & $12.5 \mathrm{a}$ & $12.5 \mathrm{a}$ & $12.2 \mathrm{a}$ & $12.2 \mathrm{a}$ & $12.6 \mathrm{a}$ \\
\hline Embrapa-122 & $12.0 \mathrm{a}$ & $12.4 \mathrm{a}$ & $12.0 \mathrm{~b}$ & $12.1 \mathrm{a}$ & $12.6 \mathrm{a}$ & $12.0 \mathrm{~b}$ & $12.0 \mathrm{a}$ & $11.9 \mathrm{~b}$ & $11.1 \mathrm{~b}$ \\
\hline Olizun-03 & $12.0 \mathrm{a}$ & $12.3 \mathrm{a}$ & $13.0 \mathrm{a}$ & $12.3 \mathrm{a}$ & $12.4 \mathrm{a}$ & $11.9 \mathrm{~b}$ & $11.6 \mathrm{a}$ & $13.7 \mathrm{a}$ & $12.4 \mathrm{a}$ \\
\hline Charrua & $11.9 \mathrm{a}$ & $12.0 \mathrm{a}$ & $11.9 \mathrm{~b}$ & $11.9 \mathrm{a}$ & $11.9 \mathrm{~b}$ & $11.5 \mathrm{~b}$ & $12.0 \mathrm{a}$ & $11.4 \mathrm{~b}$ & $11.1 \mathrm{~b}$ \\
\hline Paraíso-55 & $11.8 \mathrm{a}$ & $12.2 \mathrm{a}$ & $11.9 \mathrm{~b}$ & $11.9 \mathrm{a}$ & $12.5 \mathrm{~b}$ & $11.9 \mathrm{~b}$ & $11.5 \mathrm{a}$ & $12.6 \mathrm{a}$ & $11.7 \mathrm{~b}$ \\
\hline BRS-324 & $11.3 \mathrm{a}$ & $11.3 \mathrm{a}$ & $11.2 \mathrm{~b}$ & $11.6 \mathrm{a}$ & $11.3 \mathrm{~b}$ & $11.4 \mathrm{~b}$ & $11.4 \mathrm{a}$ & $10.2 \mathrm{~b}$ & $10.4 \mathrm{~b}$ \\
\hline M734 & $11.0 \mathrm{a}$ & $11.2 \mathrm{a}$ & $10.8 \mathrm{~b}$ & $11.1 \mathrm{a}$ & $11.0 \mathrm{~b}$ & $11.0 \mathrm{~b}$ & $11.0 \mathrm{a}$ & $11.8 \mathrm{~b}$ & $11.3 \mathrm{~b}$ \\
\hline
\end{tabular}

${ }^{1}$ For each sample size, averages followed by the same letter do not differ between themselves at $5 \%$ probability by the Scott-Knott test(1974)

evaluated from one row (Table 4), and with sample sizes of 18,10 and 02 (disagreeing with the F-test results, which indicated no effect from the cultivars for sample size 2), when the plants were evaluated from the two rows of the useable area (Table 6). Discrepancies may occur between the F-test and the various multiple-comparison tests, occurring more often with some tests than with others (SOUSA; LIRA JÚNIOR; FERREIRA, 2012).

Statistical tests require that error distribution be normal. A desirable characteristic for a test is that when 
this assumption is untrue, the test shows control of error rates for types I and II. This type of characteristic is known as the robustness of the procedure (BORGES; FERREIRA, 2003). The F-test is considered a robust test with low sensitivity to problems of non-compliance of the data to the prerequisites of variance analysis (MOORE, 2000). As a complement to the F-test, a test for the multiple comparison of averages is needed, which shows the same consistency of results, and is in agreement with those results returned by the F-test (SOUSA; LIRA

Table 5 - Averages of stem diameter and plant height in sunflower cultivars evaluated by sample size in one row of plants from plots with two rows

\begin{tabular}{|c|c|c|c|c|c|c|c|c|c|}
\hline \multirow{3}{*}{ Cultivar } & \multicolumn{9}{|c|}{ Sample size (number of sampling units) } \\
\hline & 18 & 16 & 14 & 12 & 10 & 8 & 6 & 4 & 2 \\
\hline & \multicolumn{9}{|c|}{ Stem diameter $(\mathrm{mm})$} \\
\hline Neon & $18.4 \mathrm{a}$ & $18.7 \mathrm{a}$ & $19.3 \mathrm{a}$ & $18.8 \mathrm{a}$ & $18.4 \mathrm{a}$ & $19.0 \mathrm{a}$ & $18.5 \mathrm{a}$ & $19.5 \mathrm{a}$ & $18.6 \mathrm{a}$ \\
\hline Aguará-05 & $18.2 \mathrm{a}$ & $18.1 \mathrm{a}$ & $18.1 \mathrm{~b}$ & $18.3 \mathrm{a}$ & $18.3 \mathrm{a}$ & $17.8 \mathrm{~b}$ & $18.4 \mathrm{a}$ & $17.6 \mathrm{~b}$ & $17.8 \mathrm{a}$ \\
\hline Olizun-03 & $18.1 \mathrm{a}$ & $18.9 \mathrm{a}$ & $19.7 \mathrm{a}$ & $18.5 \mathrm{a}$ & $19.1 \mathrm{a}$ & $18.8 \mathrm{a}$ & $18.2 \mathrm{a}$ & $19.1 \mathrm{a}$ & $16.8 \mathrm{a}$ \\
\hline Charrua & $17.4 \mathrm{a}$ & $18.0 \mathrm{a}$ & $17.7 \mathrm{~b}$ & $17.4 \mathrm{~b}$ & $17.8 \mathrm{a}$ & $17.2 \mathrm{~b}$ & $17.5 \mathrm{a}$ & $16.4 \mathrm{c}$ & $16.9 \mathrm{a}$ \\
\hline Zenit & $17.3 \mathrm{a}$ & $17.4 \mathrm{a}$ & $17.4 \mathrm{~b}$ & $17.2 \mathrm{~b}$ & $17.8 \mathrm{a}$ & $17.5 \mathrm{~b}$ & $16.6 \mathrm{a}$ & $17.2 \mathrm{~b}$ & $16.7 \mathrm{a}$ \\
\hline Aguará-03 & $17.3 \mathrm{a}$ & $17.3 \mathrm{a}$ & $17.7 \mathrm{~b}$ & $17.2 \mathrm{~b}$ & $17.2 \mathrm{a}$ & $17.2 \mathrm{~b}$ & $17.4 \mathrm{a}$ & $17.6 \mathrm{~b}$ & $18.4 \mathrm{a}$ \\
\hline Embrapa-122 & $17.1 \mathrm{a}$ & $16.5 \mathrm{~b}$ & $17.2 \mathrm{~b}$ & $16.9 \mathrm{~b}$ & $16.9 \mathrm{a}$ & $16.8 \mathrm{~b}$ & $16.9 \mathrm{a}$ & $17.6 \mathrm{~b}$ & $17.0 \mathrm{a}$ \\
\hline Paraíso-65 & $16.9 \mathrm{a}$ & $18.2 \mathrm{a}$ & $17.8 \mathrm{~b}$ & $16.7 \mathrm{~b}$ & $17.2 \mathrm{a}$ & $17.5 \mathrm{~b}$ & $17.5 \mathrm{a}$ & $18.0 \mathrm{~b}$ & $17.2 \mathrm{a}$ \\
\hline Aguará-06 & $16.9 \mathrm{a}$ & $17.0 \mathrm{a}$ & $17.1 \mathrm{~b}$ & $16.6 \mathrm{~b}$ & $17.1 \mathrm{a}$ & $16.9 \mathrm{~b}$ & $16.7 \mathrm{a}$ & $16.8 \mathrm{~b}$ & $17.1 \mathrm{a}$ \\
\hline Aguará-04 & $15.8 \mathrm{~b}$ & $16.3 \mathrm{~b}$ & $16.2 \mathrm{c}$ & $16.0 \mathrm{~b}$ & $15.9 \mathrm{c}$ & $16.1 \mathrm{c}$ & $15.7 \mathrm{~b}$ & $16.5 \mathrm{c}$ & $15.6 \mathrm{~b}$ \\
\hline Paraíso103CL & $15.8 \mathrm{~b}$ & $16.1 \mathrm{~b}$ & $15.9 \mathrm{c}$ & $15.9 \mathrm{~b}$ & $15.7 \mathrm{c}$ & $16.1 \mathrm{c}$ & $16.0 \mathrm{~b}$ & $16.1 \mathrm{c}$ & $15.8 \mathrm{~b}$ \\
\hline M734 & $15.7 \mathrm{~b}$ & $15.8 \mathrm{~b}$ & $15.5 \mathrm{c}$ & $16.2 \mathrm{~b}$ & $15.8 \mathrm{c}$ & $15.2 \mathrm{c}$ & $15.8 \mathrm{~b}$ & $15.7 \mathrm{c}$ & $15.3 \mathrm{~b}$ \\
\hline BRS-324 & $15.1 \mathrm{~b}$ & $15.4 \mathrm{~b}$ & $15.0 \mathrm{c}$ & $15.5 \mathrm{~b}$ & $14.5 \mathrm{~d}$ & $15.2 \mathrm{c}$ & $14.8 \mathrm{~b}$ & $15.1 \mathrm{~d}$ & $15.6 \mathrm{~b}$ \\
\hline Paraíso-55 & $14.1 \mathrm{c}$ & $14.1 \mathrm{c}$ & $13.8 \mathrm{~d}$ & $13.8 \mathrm{c}$ & $13.9 \mathrm{~d}$ & $14.3 \mathrm{~d}$ & $13.9 \mathrm{c}$ & $14.0 \mathrm{~d}$ & $14.0 \mathrm{c}$ \\
\hline BRS-321 & $13.2 \mathrm{c}$ & $12.9 \mathrm{c}$ & $12.6 \mathrm{~d}$ & $13.0 \mathrm{c}$ & $13.2 \mathrm{~d}$ & $13.3 \mathrm{~d}$ & $12.8 \mathrm{c}$ & $13.9 \mathrm{~d}$ & $13.1 \mathrm{c}$ \\
\hline Cultivar & \multicolumn{9}{|c|}{ Plant height $(\mathrm{cm})$} \\
\hline Aguará-06 & $145 \mathrm{a}$ & $145 \mathrm{a}$ & $147 \mathrm{a}$ & $146 \mathrm{a}$ & $145 \mathrm{a}$ & $144 \mathrm{a}$ & $145 \mathrm{a}$ & $146 \mathrm{a}$ & $147 \mathrm{a}$ \\
\hline Aguará-05 & $144 \mathrm{a}$ & $142 \mathrm{a}$ & $143 \mathrm{a}$ & $145 \mathrm{a}$ & $144 \mathrm{a}$ & $142 \mathrm{a}$ & $147 \mathrm{a}$ & $140 \mathrm{a}$ & $143 \mathrm{a}$ \\
\hline Neon & $140 \mathrm{a}$ & $140 \mathrm{a}$ & $141 \mathrm{a}$ & $141 \mathrm{a}$ & $137 \mathrm{~b}$ & $138 \mathrm{a}$ & $140 \mathrm{a}$ & $140 \mathrm{a}$ & $141 \mathrm{a}$ \\
\hline Aguará-04 & $135 \mathrm{~b}$ & $138 \mathrm{a}$ & $137 \mathrm{a}$ & $136 \mathrm{a}$ & $137 \mathrm{~b}$ & $138 \mathrm{a}$ & $136 \mathrm{~b}$ & $137 \mathrm{a}$ & $136 \mathrm{a}$ \\
\hline Aguará-03 & $135 \mathrm{~b}$ & $134 \mathrm{~b}$ & $132 \mathrm{~b}$ & $134 \mathrm{~b}$ & $134 \mathrm{~b}$ & $131 \mathrm{~b}$ & $133 \mathrm{~b}$ & $131 \mathrm{a}$ & $137 \mathrm{a}$ \\
\hline Embrapa-122 & $135 \mathrm{~b}$ & $134 \mathrm{~b}$ & $138 \mathrm{a}$ & $137 \mathrm{a}$ & $136 \mathrm{~b}$ & $135 \mathrm{~b}$ & $134 \mathrm{~b}$ & $137 \mathrm{a}$ & $130 \mathrm{~b}$ \\
\hline Olizun-03 & $135 \mathrm{~b}$ & $137 \mathrm{a}$ & $137 \mathrm{a}$ & $137 \mathrm{a}$ & $136 \mathrm{~b}$ & $136 \mathrm{a}$ & $132 \mathrm{~b}$ & $138 \mathrm{a}$ & $132 \mathrm{~b}$ \\
\hline Charrua & $133 \mathrm{~b}$ & $134 \mathrm{~b}$ & $131 \mathrm{~b}$ & $133 \mathrm{~b}$ & $135 \mathrm{~b}$ & $132 \mathrm{~b}$ & $132 \mathrm{~b}$ & $130 \mathrm{a}$ & $128 \mathrm{~b}$ \\
\hline Zenit & $126 \mathrm{~b}$ & $127 \mathrm{~b}$ & $126 \mathrm{~b}$ & $127 \mathrm{~b}$ & $127 \mathrm{c}$ & $131 \mathrm{~b}$ & $124 \mathrm{c}$ & $124 \mathrm{~b}$ & $128 \mathrm{~b}$ \\
\hline Paraíso-65 & $124 \mathrm{c}$ & $127 \mathrm{~b}$ & $127 \mathrm{~b}$ & $125 \mathrm{~b}$ & $126 \mathrm{c}$ & $126 \mathrm{~b}$ & $127 \mathrm{~b}$ & $127 \mathrm{a}$ & $129 \mathrm{~b}$ \\
\hline BRS-324 & $120 \mathrm{c}$ & $119 \mathrm{c}$ & $117 \mathrm{c}$ & $120 \mathrm{c}$ & $115 \mathrm{~d}$ & $122 \mathrm{c}$ & $116 \mathrm{c}$ & $118 \mathrm{~b}$ & $117 \mathrm{c}$ \\
\hline M734 & $115 \mathrm{~d}$ & $114 \mathrm{c}$ & $114 \mathrm{c}$ & $116 \mathrm{c}$ & $113 \mathrm{~d}$ & $112 \mathrm{~d}$ & $116 \mathrm{c}$ & $119 \mathrm{~b}$ & $113 \mathrm{c}$ \\
\hline BRS-321 & $109 \mathrm{~d}$ & $108 \mathrm{~d}$ & $107 \mathrm{~d}$ & $110 \mathrm{~d}$ & $109 \mathrm{~d}$ & $109 \mathrm{~d}$ & $109 \mathrm{~d}$ & $113 \mathrm{~b}$ & $105 \mathrm{c}$ \\
\hline Paraíso103CL & $109 \mathrm{~d}$ & $110 \mathrm{~d}$ & $108 \mathrm{~d}$ & $109 \mathrm{~d}$ & $109 \mathrm{~d}$ & $108 \mathrm{~d}$ & $111 \mathrm{~d}$ & $110 \mathrm{~b}$ & $111 \mathrm{c}$ \\
\hline Paraíso-55 & $107 \mathrm{~d}$ & $106 \mathrm{~d}$ & $105 \mathrm{~d}$ & $105 \mathrm{~d}$ & $105 \mathrm{~d}$ & $107 \mathrm{~d}$ & $106 \mathrm{~d}$ & $109 \mathrm{~b}$ & $106 \mathrm{c}$ \\
\hline
\end{tabular}

${ }^{1}$ For each sample size, averages followed by the same letter do not differ between themselves at $5 \%$ probability by the Scott-Knott test(1974) 
JÚNIOR; FERREIRA, 2012). Borges and Ferreira (2003) showed that the Scott-Knott test (1974) is robust and was more powerful than other multiple comparison tests. The discrepancies seen between the F-test and the Scott-Knott test (1974) in the evaluation of head diameter may therefore indicate problems in the evaluation of this characteristic, including imprecise measurements.

Except for head diameter when evaluated for 2 plants, and stem diameter when evaluated for 2-6 plants, there were no significant differences between the sampling

Table 6 - Averages of number of leaves and head diameter in sunflower cultivars evaluated by sample size in two rows of plants from plots with two rows

\begin{tabular}{|c|c|c|c|c|c|c|c|c|c|}
\hline \multirow{3}{*}{ Cultivar } & \multicolumn{9}{|c|}{ Sample size (number of sampling units) } \\
\hline & 18 & 16 & 14 & 12 & 10 & 8 & 6 & 4 & 2 \\
\hline & \multicolumn{9}{|c|}{ Number of leaves plant ${ }^{-1}$} \\
\hline Aguará-05 & $28.1 \mathrm{a}$ & $28.3 \mathrm{a}$ & $28.5 \mathrm{a}$ & $28.8 \mathrm{a}$ & $28.9 \mathrm{a}$ & $28.5 \mathrm{a}$ & $28.7 \mathrm{a}$ & $28.9 \mathrm{a}$ & $29.9 \mathrm{a}$ \\
\hline Aguará-03 & $27.7 \mathrm{a}$ & $27.5 \mathrm{a}$ & $27.6 \mathrm{a}$ & $27.4 \mathrm{a}$ & $27.6 \mathrm{a}$ & $27.3 \mathrm{a}$ & $27.8 \mathrm{a}$ & $27.2 \mathrm{a}$ & $28.2 \mathrm{a}$ \\
\hline Aguará-06 & $27.2 \mathrm{a}$ & $27.2 \mathrm{a}$ & $27.3 \mathrm{a}$ & $27.4 \mathrm{a}$ & $28.2 \mathrm{a}$ & $27.6 \mathrm{a}$ & $27.0 \mathrm{a}$ & $27.6 \mathrm{a}$ & $27.6 \mathrm{a}$ \\
\hline Charrua & $26.6 \mathrm{~b}$ & $26.6 \mathrm{a}$ & $27.1 \mathrm{a}$ & $27.0 \mathrm{a}$ & $27.3 \mathrm{a}$ & $27.0 \mathrm{a}$ & $28.8 \mathrm{a}$ & $26.4 \mathrm{a}$ & $26.7 \mathrm{~b}$ \\
\hline Olizun-03 & $26.2 \mathrm{~b}$ & $27.1 \mathrm{a}$ & $27.3 \mathrm{a}$ & $26.9 \mathrm{a}$ & $27.3 \mathrm{a}$ & $26.8 \mathrm{a}$ & $27.7 \mathrm{a}$ & $27.2 \mathrm{a}$ & $27.2 \mathrm{a}$ \\
\hline Aguará-04 & $26.2 \mathrm{~b}$ & $26.5 \mathrm{a}$ & $26.8 \mathrm{a}$ & $27.1 \mathrm{a}$ & $26.7 \mathrm{a}$ & $27.1 \mathrm{a}$ & $27.1 \mathrm{a}$ & $27.4 \mathrm{a}$ & $26.1 \mathrm{~b}$ \\
\hline Embrapa-122 & $24.9 \mathrm{~b}$ & $24.9 \mathrm{~b}$ & $24.6 \mathrm{~b}$ & $23.9 \mathrm{~b}$ & $24.5 \mathrm{~b}$ & $24.6 \mathrm{~b}$ & $23.9 \mathrm{c}$ & $23.6 \mathrm{c}$ & $25.6 \mathrm{~b}$ \\
\hline Paraíso-65 & $23.7 \mathrm{c}$ & $23.7 \mathrm{c}$ & $23.9 \mathrm{~b}$ & $23.7 \mathrm{~b}$ & $23.2 \mathrm{~b}$ & $23.8 \mathrm{c}$ & $24.7 \mathrm{~b}$ & $24.7 \mathrm{~b}$ & $24.8 \mathrm{~b}$ \\
\hline Neon & $23.2 \mathrm{c}$ & $22.4 \mathrm{c}$ & $23.6 \mathrm{~b}$ & $23.4 \mathrm{~b}$ & $23.3 \mathrm{~b}$ & $22.7 \mathrm{c}$ & $22.9 \mathrm{c}$ & $23.8 \mathrm{c}$ & $23.7 \mathrm{c}$ \\
\hline BRS-321 & $22.5 \mathrm{c}$ & $22.5 \mathrm{c}$ & $22.6 \mathrm{c}$ & $22.4 \mathrm{c}$ & $22.8 \mathrm{~b}$ & $22.3 \mathrm{c}$ & $22.6 \mathrm{c}$ & $21.6 \mathrm{c}$ & $22.4 \mathrm{c}$ \\
\hline BRS-324 & $22.5 \mathrm{c}$ & $22.5 \mathrm{c}$ & $22.8 \mathrm{c}$ & $22.6 \mathrm{c}$ & $22.3 \mathrm{~b}$ & $21.9 \mathrm{c}$ & $22.3 \mathrm{c}$ & $22.5 \mathrm{c}$ & $22.0 \mathrm{c}$ \\
\hline Zenit & $22.0 \mathrm{c}$ & $21.9 \mathrm{c}$ & $22.1 \mathrm{c}$ & $22.0 \mathrm{c}$ & $22.3 \mathrm{~b}$ & $22.3 \mathrm{c}$ & $22.3 \mathrm{c}$ & $22.5 \mathrm{c}$ & $22.3 \mathrm{c}$ \\
\hline Paraíso-55 & $20.7 \mathrm{~d}$ & $20.8 \mathrm{~d}$ & $20.8 \mathrm{~d}$ & $20.9 \mathrm{~d}$ & $20.8 \mathrm{c}$ & $21.5 \mathrm{c}$ & $21.1 \mathrm{~d}$ & $20.9 \mathrm{~d}$ & $21.3 \mathrm{c}$ \\
\hline M734 & $20.5 \mathrm{~d}$ & $20.1 \mathrm{~d}$ & $20.2 \mathrm{~d}$ & $20.2 \mathrm{~d}$ & $20.2 \mathrm{c}$ & $20.2 \mathrm{~d}$ & $20.0 \mathrm{~d}$ & $20.0 \mathrm{~d}$ & $20.4 \mathrm{c}$ \\
\hline Paraíso103CL & $19.8 \mathrm{~d}$ & $19.7 \mathrm{~d}$ & $19.5 \mathrm{~d}$ & $19.4 \mathrm{~d}$ & $20.1 \mathrm{c}$ & $19.8 \mathrm{~d}$ & $20.1 \mathrm{~d}$ & $18.7 \mathrm{~d}$ & $20.1 \mathrm{c}$ \\
\hline Cultivar & \multicolumn{9}{|c|}{ Head diameter $(\mathrm{cm})$} \\
\hline Paraíso-65 & $13.1 \mathrm{a}$ & $12.9 \mathrm{a}$ & $13.1 \mathrm{a}$ & $13.2 \mathrm{a}$ & $13.2 \mathrm{a}$ & $13.9 \mathrm{a}$ & $13.8 \mathrm{a}$ & $13.4 \mathrm{a}$ & $13.7 \mathrm{a}$ \\
\hline Zenit & $12.8 \mathrm{a}$ & $12.5 \mathrm{a}$ & $12.9 \mathrm{a}$ & $12.8 \mathrm{a}$ & $12.5 \mathrm{a}$ & $12.6 \mathrm{a}$ & $13.1 \mathrm{a}$ & $12.8 \mathrm{a}$ & $12.6 \mathrm{a}$ \\
\hline Aguará-06 & $12.7 \mathrm{a}$ & $13.1 \mathrm{a}$ & $12.8 \mathrm{a}$ & $13.1 \mathrm{a}$ & $13.0 \mathrm{a}$ & $13.5 \mathrm{a}$ & $12.6 \mathrm{a}$ & $13.8 \mathrm{a}$ & $13.1 \mathrm{a}$ \\
\hline Neon & $12.6 \mathrm{a}$ & $12.9 \mathrm{a}$ & $12.8 \mathrm{a}$ & $12.8 \mathrm{a}$ & $12.7 \mathrm{a}$ & $12.3 \mathrm{~b}$ & $12.7 \mathrm{a}$ & $12.6 \mathrm{a}$ & $12.8 \mathrm{a}$ \\
\hline Aguará-04 & $12.5 \mathrm{a}$ & $12.4 \mathrm{a}$ & $12.5 \mathrm{a}$ & $12.6 \mathrm{a}$ & $12.4 \mathrm{a}$ & $12.8 \mathrm{a}$ & $12.5 \mathrm{a}$ & $12.4 \mathrm{a}$ & $12.7 \mathrm{a}$ \\
\hline Olizun-03 & $12.2 \mathrm{a}$ & $12.1 \mathrm{~b}$ & $12.1 \mathrm{~b}$ & $12.4 \mathrm{a}$ & $12.3 \mathrm{a}$ & $12.3 \mathrm{~b}$ & $12.6 \mathrm{a}$ & $11.4 \mathrm{~b}$ & $12.5 \mathrm{a}$ \\
\hline Aguará-05 & $12.2 \mathrm{a}$ & $12.3 \mathrm{a}$ & $12.1 \mathrm{~b}$ & $11.8 \mathrm{c}$ & $12.8 \mathrm{a}$ & $12.2 \mathrm{~b}$ & $12.4 \mathrm{a}$ & $12.4 \mathrm{a}$ & $12.2 \mathrm{a}$ \\
\hline Paraíso103CL & $12.1 \mathrm{a}$ & $12.2 \mathrm{a}$ & $12.2 \mathrm{~b}$ & $12.2 \mathrm{~b}$ & $12.4 \mathrm{a}$ & $12.2 \mathrm{~b}$ & $12.3 \mathrm{a}$ & $12.0 \mathrm{~b}$ & $11.5 \mathrm{a}$ \\
\hline Embrapa-122 & $12.1 \mathrm{a}$ & $11.9 \mathrm{~b}$ & $11.9 \mathrm{~b}$ & $11.9 \mathrm{~b}$ & $12.3 \mathrm{a}$ & $11.9 \mathrm{~b}$ & $12.3 \mathrm{a}$ & $11.0 \mathrm{~b}$ & $13.3 \mathrm{a}$ \\
\hline Aguará-03 & $12.1 \mathrm{a}$ & $12.0 \mathrm{~b}$ & $12.0 \mathrm{~b}$ & $12.2 \mathrm{~b}$ & $12.2 \mathrm{a}$ & $11.8 \mathrm{~b}$ & $12.4 \mathrm{a}$ & $11.9 \mathrm{~b}$ & $13.4 \mathrm{a}$ \\
\hline Paraíso-55 & $12.1 \mathrm{a}$ & $11.8 \mathrm{~b}$ & $11.8 \mathrm{~b}$ & $11.7 \mathrm{~b}$ & $11.9 \mathrm{a}$ & $12.1 \mathrm{~b}$ & $12.1 \mathrm{a}$ & $11.7 \mathrm{~b}$ & $11.6 \mathrm{a}$ \\
\hline Charrua & $12.0 \mathrm{a}$ & $11.5 \mathrm{~b}$ & $11.8 \mathrm{~b}$ & $11.9 \mathrm{~b}$ & $11.8 \mathrm{a}$ & $11.9 \mathrm{~b}$ & $11.5 \mathrm{~b}$ & $11.6 \mathrm{~b}$ & $11.7 \mathrm{a}$ \\
\hline BRS-321 & $12.0 \mathrm{a}$ & $11.7 \mathrm{~b}$ & $12.3 \mathrm{a}$ & $11.9 \mathrm{~b}$ & $12.2 \mathrm{a}$ & $12.3 \mathrm{~b}$ & $12.3 \mathrm{a}$ & $11.0 \mathrm{~b}$ & $11.9 \mathrm{a}$ \\
\hline M734 & $11.2 \mathrm{a}$ & $10.9 \mathrm{~b}$ & $10.9 \mathrm{~b}$ & $11.0 \mathrm{~b}$ & $11.0 \mathrm{a}$ & $11.1 \mathrm{~b}$ & $11.1 \mathrm{~b}$ & $10.8 \mathrm{~b}$ & $10.8 \mathrm{a}$ \\
\hline BRS-324 & $10.9 \mathrm{a}$ & $11.0 \mathrm{~b}$ & $11.1 \mathrm{~b}$ & $11.5 \mathrm{~b}$ & $11.2 \mathrm{a}$ & $11.0 \mathrm{~b}$ & $11.0 \mathrm{~b}$ & $11.5 \mathrm{~b}$ & $12.0 \mathrm{a}$ \\
\hline
\end{tabular}

${ }^{1}$ For each sample size, averages followed by the same letter do not differ between themselves at $5 \%$ probability by the Scott-Knott test(1974) 
methods used in the present work, as regards the magnitude of the experimental coefficient of variation (differences of more than two percentage points) (Table 8).

Gomes et al. (1963) concluded that for five characteristics of sugar cane, it was more appropriate to use samples made up of a fixed number of stems per plot, one from each clump, and spread throughout the area of the plot, than to collect nearby stalks or those from the same clump. Silva et al. (1993) when estimating three characteristics of corn, found it to be more advantageous

Table 7 - Averages of stem diameter and plant height in sunflower cultivars evaluated by sample size in two rows of plants from plots with two rows

\begin{tabular}{|c|c|c|c|c|c|c|c|c|c|}
\hline \multirow{3}{*}{ Cultivar } & \multicolumn{9}{|c|}{ Sample size (number of sampling units) } \\
\hline & 18 & 16 & 14 & 12 & 10 & 8 & 6 & 4 & 2 \\
\hline & \multicolumn{9}{|c|}{ Stem diameter $(\mathrm{mm})$} \\
\hline Olizun-03 & $18.8 \mathrm{a}$ & $18.7 \mathrm{a}$ & $18.8 \mathrm{a}$ & $18.7 \mathrm{a}$ & $19.5 \mathrm{a}$ & $18.7 \mathrm{a}$ & $19.0 \mathrm{a}$ & $19.2 \mathrm{a}$ & $19.3 \mathrm{a}$ \\
\hline Neon & $18.7 \mathrm{a}$ & $18.7 \mathrm{a}$ & $19.1 \mathrm{a}$ & $19.1 \mathrm{a}$ & $18.9 \mathrm{a}$ & $18.6 \mathrm{a}$ & $19.7 \mathrm{a}$ & $19.3 \mathrm{a}$ & $18.9 \mathrm{a}$ \\
\hline Aguará-05 & $17.9 \mathrm{a}$ & $18.0 \mathrm{a}$ & $18.4 \mathrm{a}$ & $18.2 \mathrm{a}$ & $18.3 \mathrm{a}$ & $18.1 \mathrm{a}$ & $18.4 \mathrm{a}$ & $17.6 \mathrm{a}$ & $19.8 \mathrm{a}$ \\
\hline Embrapa-122 & $17.7 \mathrm{a}$ & $16.7 \mathrm{~b}$ & $17.0 \mathrm{~b}$ & $16.8 \mathrm{a}$ & $17.3 \mathrm{~b}$ & $17.2 \mathrm{a}$ & $16.7 \mathrm{~b}$ & $16.5 \mathrm{a}$ & $16.9 \mathrm{~b}$ \\
\hline Paraíso-65 & $17.7 \mathrm{a}$ & $17.4 \mathrm{~b}$ & $17.8 \mathrm{~b}$ & $17.7 \mathrm{a}$ & $18.0 \mathrm{~b}$ & $18.0 \mathrm{a}$ & $17.9 \mathrm{~b}$ & $17.9 \mathrm{a}$ & $17.7 \mathrm{~b}$ \\
\hline Aguará-03 & $17.5 \mathrm{a}$ & $17.1 \mathrm{~b}$ & $17.3 \mathrm{~b}$ & $17.8 \mathrm{a}$ & $17.7 \mathrm{~b}$ & $17.8 \mathrm{a}$ & $17.6 \mathrm{~b}$ & $16.9 \mathrm{a}$ & $19.2 \mathrm{a}$ \\
\hline Zenit & $17.4 \mathrm{a}$ & $17.4 \mathrm{~b}$ & $17.3 \mathrm{~b}$ & $17.3 \mathrm{a}$ & $17.3 \mathrm{~b}$ & $17.1 \mathrm{a}$ & $17.9 \mathrm{~b}$ & $17.8 \mathrm{a}$ & $17.0 \mathrm{~b}$ \\
\hline Charrua & $17.3 \mathrm{a}$ & $17.1 \mathrm{~b}$ & $17.6 \mathrm{~b}$ & $17.8 \mathrm{a}$ & $17.4 \mathrm{~b}$ & $17.7 \mathrm{a}$ & $17.3 \mathrm{~b}$ & $16.8 \mathrm{a}$ & $17.4 \mathrm{~b}$ \\
\hline Aguará-06 & $16.6 \mathrm{a}$ & $17.2 \mathrm{~b}$ & $16.9 \mathrm{~b}$ & $17.1 \mathrm{a}$ & $16.9 \mathrm{~b}$ & $17.0 \mathrm{a}$ & $16.9 \mathrm{~b}$ & $17.3 \mathrm{a}$ & $16.8 \mathrm{~b}$ \\
\hline Aguará-04 & $16.3 \mathrm{a}$ & $15.9 \mathrm{c}$ & $16.3 \mathrm{c}$ & $16.1 \mathrm{~b}$ & $16.2 \mathrm{c}$ & $16.1 \mathrm{~b}$ & $16.4 \mathrm{~b}$ & $16.4 \mathrm{a}$ & $15.5 \mathrm{c}$ \\
\hline M734 & $15.7 \mathrm{~b}$ & $15.7 \mathrm{c}$ & $15.4 \mathrm{c}$ & $15.6 \mathrm{~b}$ & $15.7 \mathrm{c}$ & $16.2 \mathrm{~b}$ & $15.0 \mathrm{c}$ & $16.0 \mathrm{a}$ & $15.8 \mathrm{c}$ \\
\hline Paraíso103CL & $15.6 \mathrm{~b}$ & $15.9 \mathrm{c}$ & $16.0 \mathrm{c}$ & $15.9 \mathrm{~b}$ & $15.9 \mathrm{c}$ & $16.0 \mathrm{~b}$ & $15.4 \mathrm{c}$ & $15.3 \mathrm{a}$ & $15.7 \mathrm{c}$ \\
\hline BRS-324 & $15.1 \mathrm{~b}$ & $14.9 \mathrm{c}$ & $15.2 \mathrm{c}$ & $15.3 \mathrm{~b}$ & $15.1 \mathrm{c}$ & $14.9 \mathrm{~b}$ & $15.1 \mathrm{c}$ & $14.7 \mathrm{a}$ & $15.9 \mathrm{c}$ \\
\hline Paraíso-55 & $14.5 \mathrm{~b}$ & $14.4 \mathrm{~d}$ & $14.7 \mathrm{~d}$ & $14.5 \mathrm{~b}$ & $14.4 \mathrm{c}$ & $15.1 \mathrm{~b}$ & $14.8 \mathrm{c}$ & $14.7 \mathrm{a}$ & $14.6 \mathrm{c}$ \\
\hline BRS-321 & $12.8 \mathrm{c}$ & $13.1 \mathrm{~d}$ & $13.1 \mathrm{~d}$ & $12.8 \mathrm{c}$ & $12.5 \mathrm{~d}$ & $13.0 \mathrm{c}$ & $13.1 \mathrm{c}$ & $15.9 \mathrm{a}$ & $12.8 \mathrm{c}$ \\
\hline Cultivar & \multicolumn{9}{|c|}{ Plant height $(\mathrm{cm})$} \\
\hline Aguará-06 & $144 \mathrm{a}$ & $146 \mathrm{a}$ & $145 \mathrm{a}$ & $146 \mathrm{a}$ & $145 \mathrm{a}$ & $145 \mathrm{a}$ & $145 \mathrm{a}$ & $144 \mathrm{a}$ & $148 \mathrm{a}$ \\
\hline Aguará-05 & $142 \mathrm{a}$ & $141 \mathrm{a}$ & $144 \mathrm{a}$ & $140 \mathrm{a}$ & $145 \mathrm{a}$ & $143 \mathrm{a}$ & $142 \mathrm{a}$ & $140 \mathrm{a}$ & $143 \mathrm{a}$ \\
\hline Neon & $140 \mathrm{a}$ & $141 \mathrm{a}$ & $142 \mathrm{a}$ & $142 \mathrm{a}$ & $140 \mathrm{a}$ & $138 \mathrm{a}$ & $141 \mathrm{a}$ & $140 \mathrm{a}$ & $144 \mathrm{a}$ \\
\hline Aguará-04 & $137 \mathrm{a}$ & $136 \mathrm{a}$ & $136 \mathrm{~b}$ & $137 \mathrm{a}$ & $138 \mathrm{a}$ & $136 \mathrm{a}$ & $138 \mathrm{a}$ & $139 \mathrm{a}$ & $133 \mathrm{~b}$ \\
\hline Olizun-03 & $136 \mathrm{a}$ & $134 \mathrm{~b}$ & $136 \mathrm{~b}$ & $133 \mathrm{~b}$ & $139 \mathrm{a}$ & $133 \mathrm{~b}$ & $137 \mathrm{a}$ & $135 \mathrm{a}$ & $141 \mathrm{a}$ \\
\hline Embrapa-122 & $134 \mathrm{~b}$ & $132 \mathrm{~b}$ & $135 \mathrm{~b}$ & $134 \mathrm{~b}$ & $137 \mathrm{a}$ & $136 \mathrm{a}$ & $135 \mathrm{a}$ & $132 \mathrm{~b}$ & $133 \mathrm{~b}$ \\
\hline Aguará-03 & $134 \mathrm{~b}$ & $134 \mathrm{~b}$ & $133 \mathrm{~b}$ & $136 \mathrm{~b}$ & $134 \mathrm{~b}$ & $133 \mathrm{~b}$ & $136 \mathrm{a}$ & $132 \mathrm{~b}$ & $136 \mathrm{~b}$ \\
\hline Charrua & $132 \mathrm{~b}$ & $137 \mathrm{a}$ & $132 \mathrm{~b}$ & $133 \mathrm{~b}$ & $133 \mathrm{~b}$ & $134 \mathrm{~b}$ & $134 \mathrm{a}$ & $129 \mathrm{~b}$ & $131 \mathrm{~b}$ \\
\hline Paraíso-65 & $127 \mathrm{~b}$ & $127 \mathrm{~b}$ & $125 \mathrm{c}$ & $126 \mathrm{~b}$ & $128 \mathrm{~b}$ & $126 \mathrm{~b}$ & $128 \mathrm{~b}$ & $124 \mathrm{~b}$ & $129 \mathrm{~b}$ \\
\hline Zenit & $126 \mathrm{~b}$ & $126 \mathrm{~b}$ & $127 \mathrm{c}$ & $125 \mathrm{~b}$ & $125 \mathrm{c}$ & $127 \mathrm{~b}$ & $127 \mathrm{~b}$ & $125 \mathrm{~b}$ & $130 \mathrm{~b}$ \\
\hline BRS-324 & $118 \mathrm{c}$ & $119 \mathrm{c}$ & $118 \mathrm{~d}$ & $122 \mathrm{c}$ & $122 \mathrm{c}$ & $118 \mathrm{c}$ & $119 \mathrm{c}$ & $117 \mathrm{c}$ & $119 \mathrm{c}$ \\
\hline M734 & $115 \mathrm{c}$ & $114 \mathrm{c}$ & $114 \mathrm{~d}$ & $115 \mathrm{c}$ & $116 \mathrm{~d}$ & $114 \mathrm{c}$ & $114 \mathrm{c}$ & $115 \mathrm{c}$ & $115 \mathrm{c}$ \\
\hline BRS-321 & $107 \mathrm{~d}$ & $107 \mathrm{~d}$ & $109 \mathrm{e}$ & $107 \mathrm{~d}$ & $110 \mathrm{~d}$ & $106 \mathrm{~d}$ & $110 \mathrm{c}$ & $106 \mathrm{~d}$ & $101 \mathrm{c}$ \\
\hline Paraíso103CL & $107 \mathrm{~d}$ & $108 \mathrm{~d}$ & $109 \mathrm{e}$ & $106 \mathrm{~d}$ & $111 \mathrm{~d}$ & $108 \mathrm{~d}$ & $107 \mathrm{c}$ & $104 \mathrm{~d}$ & $112 \mathrm{c}$ \\
\hline Paraíso-55 & $105 \mathrm{~d}$ & $105 \mathrm{~d}$ & $106 \mathrm{e}$ & $105 \mathrm{~d}$ & $107 \mathrm{~d}$ & $106 \mathrm{~d}$ & $107 \mathrm{c}$ & $92 \mathrm{~d}$ & $108 \mathrm{c}$ \\
\hline
\end{tabular}

${ }^{1}$ For each sample size, averages followed by the same letter do not differ between themselves at $5 \%$ probability by the Scott-Knott test(1974) 
to evaluate plants from different holes than plants from the same hole. In the present work, the evaluation of plants from the same row may have been quite similar to the evaluation of plants from two rows because the sunflowers were grown with one plant per hole. Competition should produce a different reduction in the growth and yield of plants from the same hole (for corn) or plants from the same clump (in the case of sugar cane). These reductions are likely to be different for different holes of the same plot due to the genetic factors of the plants from each hole or clump, and to the environmental factors (mainly the soil). Sampling plants from different holes should therefore encourage greater representation of the holes from each plot than would a sample of plants from the same hole.

For the two sampling methods, the experimental accuracy, as measured by the experimental coefficient of variation $(\mathrm{CV})$, increased with an increase in sample size (Table 8). When, in a random block experiment with "r" replicates, "a" sampling units are extracted per plot, then the variance estimate for a treatment mean $\left(\mathrm{s}^{2} \mathrm{x}\right)$ is $\mathrm{s}^{2} / \mathrm{ra}$, where $\mathrm{s}^{2} / \mathrm{r}$ estimates the true variance of an individual plot, plus the true variance of the mean for an individual plot or $\sigma^{2} \mathrm{a} / \mathrm{r}$ (LeCLERG; LEONARD; CLARK, 1966). In these expressions, $\mathrm{s}^{2}$ is the mean square of the residue and $\sigma^{2}$ is the true variance for an individual sampling unit taken from a plot. I.e., $\mathrm{s}^{2} / \mathrm{ra}$ estimates $\sigma^{2} / \mathrm{r}+\sigma^{2} \mathrm{a} / \mathrm{ra}$ or $1 / \mathrm{r}\left(\sigma^{2}+\sigma^{2} \mathrm{a} / \mathrm{a}\right)$. In this formula, it becomes evident that the variance of a treatment mean is influenced by the number of replicates ( $r$ ) and by the number of sampling units taken at each plot (a) (LeCLERG; LEONARD; CLARK, 1966).
Based on the data in Table 8, a sample can be recommended made up of ten sampling units for the evaluation of the number of leaves, the head diameter, the stem diameter and the plant height of sunflower cultivars. From this sample size upward, no additional gains in experimental precision are obtained. This sample size can be used to evaluate plants in a single row or in two rows taken from the useable area of each plot, since there was virtually no difference between sampling methods with regard to experimental accuracy. However, it would appear to be more practical and quicker to take samples from a single row of plants.

It is important to mention that from three (MARTIN et al., 2012) to ten (AHMAD; AHMED; TAHIR, 2012; REHMAN et al., 2012) plants were used to evaluate the height of the sunflower, from five (AMORIN et al., 2007 ; PIVETTA et al., 2012) to 20 (SMIRDELE; MOURÃO JÚNIOR; GIANLUPPI, 2005) stems were used to estimate the stem diameter from the same crop, from three (MARTIN et al., 2012) to 30 (SMIRDELE; MOURÃO JÚNIOR; GIANLUPPI, 2005) heads were used to determine the head diameter in the sunflower, and from three (MARTIN et al., 2012) to ten (AHMAD; AHMED; TAHIR, 2012; VOGT; B ALBINOT JÚNIOR; SOUZA, 2010) sunflower plants were used to evaluate the number of leaves per plant in the crop. However several authors (AHMAD; AHMED; TAHIR et al., 2012, REHMAN et al., 2012; VOGT; BALBINOT JÚNIOR; SOUZA, 2010) used ten plants to estimate the above characteristics.

Table 8 - Values for the experimental coefficient of variation in sunflower cultivars evaluated by sample size and sampling method

\begin{tabular}{|c|c|c|c|c|c|c|c|c|}
\hline \multirow{4}{*}{$\begin{array}{l}\text { Sample size } \\
\text { (sampling units) }\end{array}$} & \multicolumn{2}{|c|}{ Number of leaves plant ${ }^{-1}$} & \multicolumn{2}{|c|}{ Head diameter $(\mathrm{cm})$} & \multicolumn{2}{|c|}{ Stem diameter $(\mathrm{mm})$} & \multicolumn{2}{|c|}{ Plant height $(\mathrm{cm})$} \\
\hline & \multicolumn{8}{|c|}{ Sampling method $^{1}$} \\
\hline & Single row & Two rows & Single row & Two rows & Single row & Two rows & Single row & Two rows \\
\hline & \multicolumn{8}{|c|}{ Experimental coeficiente of variation $(\%)$} \\
\hline 2 & 11.7 & 12.5 & 17.0 & 19.9 & 17.8 & 14.2 & 11.1 & 9.8 \\
\hline 4 & 8.6 & 9.4 & 14.7 & 14.1 & 19.5 & 12.4 & 10.1 & 10.3 \\
\hline 6 & 7.2 & 8.2 & 12.9 & 13.3 & 13.1 & 11.2 & 9.1 & 8.8 \\
\hline 8 & 8.4 & 7.8 & 12.3 & 11.4 & 11.7 & 10.4 & 8.3 & 7.8 \\
\hline 10 & 7.2 & 7.0 & 11.3 & 10.6 & 11.0 & 10.0 & 7.6 & 7.6 \\
\hline 12 & 6.9 & 7.7 & 10.2 & 10.9 & 10.4 & 10.6 & 8.2 & 8.3 \\
\hline 14 & 6.5 & 7.8 & 9.9 & 10.7 & 10.3 & 10.6 & 7.9 & 8.5 \\
\hline 16 & 7.1 & 7.5 & 10.4 & 10.9 & 9.5 & 10.1 & 8.1 & 7.8 \\
\hline 18 & 7.4 & 7.6 & 10.4 & 10.1 & 10.2 & 9.1 & 8.2 & 7.7 \\
\hline
\end{tabular}

${ }^{1}$ Single row: characteristics evaluated in plants from one row in plots with two rows; two rows: half of the sampling units evaluated from one row and the remainder from the other. Rows and plants were selected randomly 


\section{CONCLUSIONS}

1. A sample made up of ten sampling units is suitable for evaluating the number of leaves, the diameters of the head and the stem, and the plant height of sunflower cultivars. From this sample size upwards no additional gains in experimental precision are obtained;

2. The sample size estimated can be used to evaluate plants in a single row or in two rows taken from the useable area of each plot, since there was virtually no difference between sampling methods with regard to experimental accuracy. However, it is most practical and quick to sample in one row of plants.

\section{REFERENCES}

AHMAD, M. W.; AHMED, M. S.; TAHIR, H. N. Combining ability analysis for achene yield and related traits in sunflower (Helianthus annuus L.). Chilean Journal of Agricultural Research, v. 72, n. 1, p. 21-26, 2012.

AMORIM, E. P. et al. Divergência genética em genótipos de girassol. Ciência e Agrotecnologia, v. 31, n. 6, p. 1637-1644, 2007.

AMJADIAN, M.; FARSHADFAR, M.; GHOLIPOOR, M. The effects of leaf removal on grain yield and yield components of sunflower, var. Alestar. Annals of Biological Research, v. 4, n. 4 , p. $42-45,2013$

BORGES, L. C.; FERREIRA, D. F. Poder e taxas de erro tipo I dos testes Scott-Knott, Tukey e Student-Newman-Keuls sob distribuições normal e não normais dos resíduos. Revista de Matemática e Estatística, v. 21, n. 1, p. 67-83, 2003.

CARGNELUTTI FILHO, A. et al. Tamanho de amostra de caracteres em híbridos de mamoneira. Ciência Rural, v. 40, n. 2 , p. $280-287,2010$.

CARGNELUTTI FILHO, A. et al. Tamanho de amostra de caracteres de cultivares de feijão. Ciência Rural, v. 38, n. 3, p. 635-642, 2008.

CARGNELUTTIFILHO,A. et al. Métodos de estimativa do tamanho ótimo de parcelas experimentais de híbridos de milho simples, triplo e duplo. Ciência Rural, v. 41, n. 9, p. 1509-1516, 2011.

CARMO FILHO, F.; OLIVEIRA, O. F. Mossoró: um município do semi-árido nordestino. Mossoró: Fundação Guimarães Duque/ ESAM, 1989. 62 p. (Col. Mossoroense, B., 672).

EMPRESA BRASILEIRA DE PESQUISA AGROPECUÁRIA. Centro Nacional de Pesquisa do Solo. Sistema Brasileiro de classificação de solos. Brasília: Serviço de Produção de Informação, 2006. 306 p.

GOMES, F. P. et al. Amostragem de cana-de-açúcar para determinações tecnológicas. Anais da E.S.A. "Luiz de Queiroz”, v. 20, p. 89-114, 1963.

LeCLERG, E. L.; LEONARD, W. H. E.; CLARK, A. G. Field plot technique. Minneapolis: Burgess, 1966. $373 \mathrm{p}$.
JAGADEESAN, S. et al. valuable sunflower dwarf mutant. Helia, v. 31, n. 49, p. 79-82, 2008.

LEITE, M. S. O. et al. Sample size for full-sib Family evaluation in sugarcane. Pesquisa Agropecuária Brasileira, v. 44, n. 12, p. 1562-1574, 2009.

MARTIN, T. N. et al. Spatial distribution of sunflower cultivars and the relationship between growth features. Revista Ciência Agronômica, v. 43, n. 2, p. 338-345, 2012.

MOORE, D. S. A estatística básica e sua prática. Rio de Janeiro: LTC - Livros Técnicos e Científicos Editora, 2000. 482 p.

PARANAIBA, P.F.; MORAIS, A. R.; FERREIRA, D. F. Tamanho ótimo de parcelas experimentais: comparação de métodos em experimentos de trigo e mandioca. Revista Brasileira de Biometria, v. 27, n. 1, p. 81-90, 2009.

PIVETTA, L. G. et al. Avaliação de híbridos de girassol e relação entre parâmetros produtivos e qualitativos. Revista Ciência Agronômica, v. 43, n. 3, p. 561-568, 2012.

REHMAN, R. et al. Using multivariate analysis for selecting desirable hybrids in sunflower (Helianthus annuus L.). Pakistan Journal of Botany, v. 44, n. 5, p. 1715-1720, 2012.

RIBEIRO JÚNIOR, J. I. Análises estatísticas no SAEG. Viçosa: Universidade Federal de Viçosa, 2001. 301 p.

SCOTT, A. J.; KNOTT, M. A. Cluster analysis method for grouping means in the analysis of variance. Biometrics, v. 30, n. 3 , p. $507-512,1974$

SILVA, A. R.; RÊGO, E. R.; CECON, P. R. Tamanho de amostra para caracterização morfológica de frutos de pimenteira. Horticultura Brasileira, v. 29, n. 1, p. 125-129, 2011.

SILVA, J.; SILVA, P. S. L.; NUNES, R. P. Amostragem e tamanho da amostra da estimação de caracteres da espiga do milho. Pesquisa Agropecuária Brasileira, v. 33, n. 12, p. 19831988, 1998

SILVA, P. S. L. et al. Métodos de amostragem e tamanho da amostra para alguns caracteres do milho. Ciência Agronômica, v. 24 , n. $1 / 2$, p. $5-10,1993$.

SMIRDELE, O. J.; MOURÃO JÚNIOR, M.; GIANLUPPI, D. Avaliação de cultivares de girassol em savana de Roraima. Acta Amazonica, v. 35, n. 3, p. 331-336, 2005.

SOUSA, C. A.; LIRA JÚNIOR, M. A.; FERREIRA, R. L. C. Avaliação de testes estatísticos de comparações múltiplas de médias. Revista Ceres, v. 59, n. 3, p. 350-354, 2012.

VOGT, G. A.; BALBINOT JÚNIOR, A. A.; SOUZA, A. M. Divergência genética entre cultivares de girassol no Planalto Norte Catarinense. Scientia Agraria, v. 11, n. 4, p. 307-315, 2010.

WOLKOWSKI, R. P.; REISDORF, T. A.; BUNDY, L. G. Field plot technique comparison for estimating corn grain and dry matter yield. Agronomy Journal, v. 80, n. 2, p. 278-280, 1988.

YASIN, A. B.; SINGH, S. Correlation and path coefficient analyses in sunflower. Journal of Plant Breeding and Crop Science, v. 2, n. 5, p. 129-133, 2010. 